Ann. Biol. anim. Bioch. Biophys., I964, 4 (I), 49-62.

\title{
LES CONSTITUANTS AZOTÉS DU LAIT DE TRUIE : ÉVOLUTION AU COURS DE LA LACTATION ET INFLUENCE DU RÉGIME ALIMENTAIRE
}

\author{
E. SALMON-LEGAGNEUR \\ avec la collaboration technique de Michelle Nocart, M. Guichard et J. Rettagliati \\ Station de Recherches sur l'Élevage des Porcs, \\ Centre national de Recherches zootechniques, Jouy-en-Josas (Seine-et-Oise)
}

\section{SOMMAIRE}

L'étude de la répartition des constituants azotés du lait de Truie, entreprise au cours de 12 lactations de truies Large White, a fait ressortir les points suivants :

$\left.\mathbf{I}^{0}\right)$ Le lait de Truie, bien que considérablement plus riche que le lait de Vache en tous les cons. tituants azotés, contient en valeur relative moins de caséine (54 p. 100) et de $\beta$-lactoglobuline ( 2 p. IOO), mais plus de lactalbumine (I4 p. Ioo), de globuline (I 8 p. Ioo) et de protéoses peptones. Par contre, les différentes fractions de l'azote non protéique diffèrent assez peu dans les deux laits.

$2^{\circ}$ ) Au cours de la lactation, les teneurs de la plupart des fractions, sauf l'urée et la créatine, baissent considérablement au début de la lactation et remontent légèrement en fin de lactation. Il s'ensuit que les quantités journalières secrétées varient peu, surtout en ce qui concerne la caséine. En valeur relative, la matière azotée du lait s'enrichit en caséine, en $\beta$-lactoglobuline et en azote non protéique au début de la lactation et s'appauvrit en toutes les autres fractions; après la première semaine, cette évolution se stabilise, sauf pour la lactalbumine qui continue à diminuer légèrement.

$\left.3^{\circ}\right)$ Un supplément alimentaire de $3^{\circ}$ p. Ioo d'azote, par rapport à un régime normal a pour effet une légère augmentation de la concentration en azote du lait, celle-ci porte principalement sur les teneurs en albumine et en urée et, à moindre degré, en caséine et en globuline.

\section{INTRODUCTION}

La composition du lait de Truie revêt une importance particulière pour la croissance du porcelet. Dans un travail récent (SALMON-LEGAGNEUR et AUmaîTRE, I962), nous avons attiré l'attention sur le rôle que pouvait jouer la richesse du lait en matière azotée comme facteur de cette croissance. Mais la composition du lait de Truie varie beaucoup et nous avons montré, qu'à côté d'autres modifications on observe 
une évolution très rapide au cours des premiers jours de lactation de certains constituants azotés et notamment de la caséine (SALMON-LEGAGNEUR, I962). Or, on sait maintenant que les différentes protéines du lait ne présentent pas la même valeur nutritionnelle. Par exemple, HENRY (I957) rapporte que la caséine a pour le Rat en croissance et le Chien des valeurs biologiques très inférieures à celles des protéines du lactosérum par suite d'un déficit en acides aminés soufrés.

Il pouvait donc paraître intéressant d'examiner plus en détail dans quelle mesure le lait de Truie pouvait présenter des variations de composition azotée propres à en modifier la valeur nutritionnelle. Or, si leš variations de la composition globale du lait de Truie sont maintenant mieux connues (NeuHaus, I96I), on ne dispose que de très peu d'informations sur la répartition des constituants azotés. Seuls SHEFFY et al. (1952) et TravNiceK (I960) ont apporté quelques précisions à ce sujet.

Dans le cadre de l'étude plus générale des facteurs de variation de la production laitière chez la Truie, nous avons voulu chercher à connaitre quelle était la répartition des principaux constituants azotés du lait de Truie et comment celle-ci évoluait. Deux aspects ont particulièrement retenu notre attention : 1'influence du stade de lactation et l'effet d'une surcharge alimentaire en protéines par rapport à un régime normal.

\section{MATÉRIEI, ET MÉTHODES}

\section{A. - Animaux}

12 truies de race Yorkshire Large White ayant déjà effectué une ou plusicurs lactations, ont été utilisées dans cette étude. Ces truies, fécondées au cours de l'été 1962 , étaient conservées pendant toute la durée de l'expérience en porcherie conditionnée $\left(20^{\circ} \mathrm{C} ; \mathrm{F}=65 \mathrm{p} .100\right)$.

Elles recevaient pendant leur gestation $2,5 \mathrm{~kg}$ par jour d'un aliment complet équilibré d'un type courant, permettant la couverture de tous les besoins, ou $5 \mathrm{~kg}$ d'un aliment enrichi en protéines (régime hyperprotidique). A cet effet, les animaux étaient répartis en $\operatorname{deux} \operatorname{lots} \mathrm{A}$ et $\mathrm{B}$ recevant l'un ou l'autre régime. La composition des différents aliments est indiquée au tableau I.

Suivant le lot, les truies recevaient donc au cours de la lactation, compte tenu de leur consommation réelle, $700 \mathrm{~g}$ de matières azotées par jour dans le lot normal et $893 \mathrm{~g}$, soit environ $30 \mathrm{p}$. roo de plus, dans le lot suralimenté.

Les lactations duraient 8 semaines pendant lesquelles les productions laitières étaient déterminées de façon hebdomadaire (SALMON-LEGAGNEUR, 1956). L'analyse des constituants azotés était pratiquée sur des échantillons individuels de lait prélevés sur chaque truie à l'aide d'une machine à traire spéciale et après injection intraveineuse à la truie de 4 UI d'ocytocine (SALMon-LEGAGNEUR, I959).

Les échantillons étaient prélevés aux dates suivantes par rapport au moment de la parturition : o heure, 24 heures, 48 heures, 7 jours, $2,3,4,5,6,7,8$ semaines. Au total i i échantillons par truie étaient donc récoltés et donnaient lieu aux déterminations suivantes : azote total, caséine, globuline, $\beta$-lactoglobuline, albumines résiduelles, protéoses-peptones, azote non protidique, urée, ammoniac acides aminés libres, créatine et créatinine. Les échantillons étaient stockés sans conservateur à $0^{\circ} \mathrm{C}$ à mesure de leur récolte et analysés par séries hebdomadaires.

On suivait, en outre, les variations de poids des truies et des porcelets au cours de l'expérience.

\section{B. - Méthodes d'analyse}

Toutes les fractions, sauf la créatine et la créatinine ont été dosées par leur azote suivant la méthode KJELDAHL et les résultats étaient exprimés en milligrammes d'azote pour $100 \mathrm{~g}$ de lait.

Pour les fractions protéiques, nous avons utilisé, en les adaptant à nos conditions, les techniques de précipitation sélective utilisées pour le lait de vache par Rowland (I938), SHahaNi (I95I) et Aschaffenburgh (1959).

Ces techniques sont conventionnelles et ne permettent pas toujours de doser les constituants 
vrais, mais seulement des fractions ainsi dénommées et elles sont en général moins précises que les techniques d'électrophorèse ou de chromatographie. Toutefois, elles présentent l'avantage de nécessiter peu de matériel, d'être aisément reproductibles et, dans notre cas, de permettre des comparaisons avec les résultats d'autres auteurs. Nous avons ainsi dosé :

- la caséine par précipitation à $\mathrm{pH} 4,6$ (Rowland, 1938),

- les globulines par précipitation par le méthanol à o' C (Shairani, 195I), 1959)

- la $\beta$-lactoglobuline après précipitation à $\mathrm{pH} 2$ des protéines du sérum (Aschaffenburg,

- l'albumine totale après précipitation à $20^{\circ} \mathrm{C}$ des autres protéines par le sulfate de soude à 20 p. IOO (ASCHAFFENBURG, I959),

- les proteoses-peptones par précipitation à pH 4,6 par le sulfate de soude à I 2 p. Io० (AsCHAFFENBURG, 1959),

- l'azote non protéique total, par précipitation à $0^{\circ} \mathrm{C}$ des protéines par l'acide trichloracétique à I 5 p. Ioo (Rowland, 1938).

Les différentes fractions de l'azote soluble ont été dosées sur les filtrats déprotéinisés, selon les techniques de Denis (IgIg), adaptées par ShaHANi (I95I), à savoir :

- l'ammoniac par distillation en présence de magnésie,

- l'urée par distillation de l'ammoniac après hydrolyse uréasique et incubation pendant $8 \mathrm{~h}$ à $40^{\circ} \mathrm{C}$,

- la créatine et la créatinine, par réaction colorée, avant ou après hydrolyse, à l'acide picrique et lecture au spectrophotomètre à $520 \mathrm{~m} \mu$,

- les acides aminés libres ont été dosés par la technique au formol de Sorensen. Bien que relativement peu précise, cette méthode offre l'avantage d'une grande simplicité et nous a donné des résultats très reproductibles en accord avec les données de SIEFFY (1952),

- enfin, la fraction restante, ou indéterminée, a été appréciée par différence. Cet azote indéterminé représente essentiellement les nucléotides et certains déchets du métabolisme azoté (acide urique, purines, glutathion).

TABLEAU I

Composition des Régimes Alimentaires.

\begin{tabular}{|c|c|c|c|}
\hline Aliments & Gestation & $\begin{array}{c}\text { Lactation } \\
\text { normal }\end{array}$ & $\begin{array}{l}\text { Lactation } \\
\text { enrichi }\end{array}$ \\
\hline$-\quad-\quad--\ldots-\cdots-$ & ---- & $-\ldots$ & - \\
\hline Orge $\ldots \ldots \ldots \ldots \ldots \ldots \ldots$ & 75 & $7 i_{t}^{\prime}$ & 6.3 \\
\hline Farine de Luzerne............. & 10 & 5 & 5 \\
\hline Son $\ldots \ldots \ldots \ldots \ldots \ldots \ldots$ & 5 & 5 & 5 \\
\hline Tourteau de soja ............ & 5 & 5 & 10 \\
\hline Farine de poisson $\ldots \ldots \ldots \ldots \ldots$ & - & 3 & 7 \\
\hline Levure................... & - & 3 & 5 \\
\hline IIsile d'arachide $. . . \ldots \ldots \ldots .$. & 2 & 2 & 2 \\
\hline Mélange minéral............. & 3 & 3 & 3 \\
\hline & 100 & 100 & 100 \\
\hline $\begin{array}{l}\text { Teneur en matière azotée totale } \\
(\mathrm{N} \times 6,25) \mathrm{g} / 100 \ldots \ldots \ldots \ldots\end{array}$ & 14,3 & 14,9 & 19,0 \\
\hline
\end{tabular}

\section{RÉSUL'TATS}

\section{A. - Répartition moyenne des constituants azotés}

Nous avons calculé pour chaque truie la teneur moyenne du lait en ses constituants azotés à partir des résultats d'analyse des différents prélèvements, abstraction faite du premier échantillon ou colostrum. Puis, à partir de ces moyennes indi- 
viduelles, nous avons calculé les moyennes et écart-types pour l'ensemble des animaux. Compte tenu du peu de différences rencontrées dans les résultats des 2 lots $A$ et $B$, ceux-ci ont été réunis et considérés ensemble. Les valeurs ainsi obtenues figurent aus tableau 2.

TABLEAU 2

Répartition des constituants azotés du lait de Truie

\begin{tabular}{|c|c|c|c|c|}
\hline & \multicolumn{2}{|c|}{$\mathrm{mg}$ de $\mathrm{N}$ p. 100 de lait } & \multicolumn{2}{|c|}{$\mathrm{N}$ p. 100 de $\mathrm{N}$ total } \\
\hline & Moyenne $\pm \sigma$ & $\begin{array}{c}\text { Coefficient de } \\
\text { variation }\end{array}$ & Moyenne $\pm \sigma$ & $\begin{array}{c}\text { Coefficient de } \\
\text { variation }\end{array}$ \\
\hline Azote total .............. & $1014,0=52,1$ & 5,1 & - & - \\
\hline Caséine................. & $545,1 \pm 39,0$ & 7,1 & $53,7 \pm 2,6$ & 4,9 \\
\hline Globuline ................. & $181,0 \pm 31,9$ & 17,6 & $17,8 \pm 2,6$ & 14,5 \\
\hline ß-lactoglobuline ............. & $19,3 \pm 5,9$ & 30,7 & $1,9 \pm 0,6$ & 31,5 \\
\hline Lactalbumine..$\ldots \ldots \ldots \ldots$ & $137,6 \pm 12,4$ & 7,9 & $13,6 \pm 1,2$ & 7,9 \\
\hline Protéoses-peptones ........... & $56,3 \pm 12,4$ & 22,0 & 5,5 土 1,3 & 20,3 \\
\hline Azote non protéique.......... & $68,3 \pm 5,0$ & 7,3 & $6,7 \pm 0,7$ & 9,7 \\
\hline Urée ..................... & $13,7 \pm 2,1$ & 15,0 & $1,3 \pm 0,1$ & 7,7 \\
\hline Ammoniac................. & $1,7 \pm 1,0$ & 62,5 & $0,2 \quad-$ & - \\
\hline Azote aminé............... & 21,9 上 2,9 & 13,4 & $2,2 \pm 0,3$ & 15,2 \\
\hline 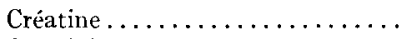 & $3,5 \pm 0,7$ & 19,8 & $0,2 \quad-$ & - \\
\hline 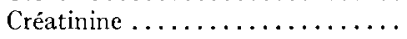 & $1,9 \pm 0,5$ & 24,2 & 0,1 & - \\
\hline Azote résiduel $\ldots \ldots \ldots \ldots \ldots$ & $28,3 \pm 7,7$ & 27,2 & $2,7 \pm 0,8$ & 9,6 \\
\hline
\end{tabular}

Ces résultats nous permettent de faire les commentaires suivants :

$\left.I^{\circ}\right)$ Comme dans beaucoup d'espèces, la caséine est le principal des constituants azotés du lait, sans toutefois atteindre, en valeur relative, une teneur aussi élevée que dans les autres laits. Il faut, par contre, souligner les teneurs élevées atteintes en valeur absolue et relative par les protéines du sérum.

$\left.2^{\circ}\right)$ Les écarts-types sont assez élevés, mais comme pour les autres constituants du lait, les variabilités sont très différentes suivant les fractions azotées. Les éléments les plus variables sont, avec l'ammoniac, les protéines du sérum, globulines et albumines, qui sont les fractions les plus difficiles à doser, et les moins variables sont la caséine et l'azote non protéique.

$\left.3^{\circ}\right)$ Il faut surtout noter la très remarquable constance dì rapport caséine/azote total, qui varie donc très peu d'un individı à l'autre (coefficient de variation : 4,9 p. IOO).

\section{B. - Evolution au cours de la lactation}

Les teneurs moyennes de chaque constituant aux différentes dates de prélèvement ont été calculées à partir de chacun des 12 résultats individuels.

Ces moyennes sont rapportées dans les tableaux 3 et 4 et dans les figures I, 2,3 et 4 . 


\section{TABLEAU 3}

Évolution des taux des constituants azotés du lait de Truie au cours de la lactation. $m g$ d'azote p. $100 \mathrm{~g}$ lait

\begin{tabular}{|c|c|c|c|c|c|c|c|c|c|c|c|}
\hline No prélèvement & 0 & 1 & 2 & 3 & 4 & 5 & 6 & 7 & 8 & 9 & 10 \\
\hline Date de prélèvement & $\begin{array}{c}0 \\
\text { Partu- } \\
\text { rition }\end{array}$ & $24 \mathrm{~h}$ & $48 \mathrm{~h}$ & $7 \mathrm{j}$ & $14 \mathrm{j}$ & $21 j$ & $28 \mathrm{j}$ & $35 \mathrm{j}$ & $42 j$ & $49 j$ & $\begin{array}{c}56 \mathrm{j} \\
\text { Sevrage }\end{array}$ \\
\hline Azote total....... & 2703 & 1688 & 1298 & 868 & 814 & 835 & 863 & 880 & 930 & 1002 & 1200 \\
\hline Caséine ........... & 846 & 701 & 621 & 481 & 464 & 465 & 492 & 510 & 540 & 576 & 707 \\
\hline Globuline & 1035 & 539 & 243 & 113 & 96 & 124 & 132 & 131 & 134 & 152 & 208 \\
\hline B-lactoglobuline ... & 35 & 40 & 26 & 22 & 20 & 20 & 16 & 13 & 9 & 13 & 15 \\
\hline Lactalbumine........ & 454 & 263 & 207 & 132 & 120 & 122 & 118 & 124 & 130 & 120 & 121 \\
\hline Protéoses-peptones... & 202 & 102 & 65 & 43 & 48 & 41 & 41 & 38 & 46 & 62 & 66 \\
\hline Azote non protéique.. & 62 & 66 & 69 & 66 & 64 & 60 & 62 & 65 & 71 & 73 & 82 \\
\hline Urée.............. & 8 & 9 & 12 & 16 & 18 & 17 & 15 & 13 & 12 & 12 & 9 \\
\hline $\mathrm{NH}_{3} \ldots \ldots \ldots \ldots$ & 0,8 & 1,2 & 1,7 & 4,3 & 2,9 & 1,4 & 1,6 & 1,1 & 1,6 & 1,1 & 0,6 \\
\hline Azote aminé ...... & 52 & 30 & 26 & 17 & 17 & 17 & 19 & 21 & 20 & 23 & 26 \\
\hline Créatine .......... & - & - & 1,5 & 2,3 & 1,9 & 2,9 & 3,9 & 4,0 & 2,5 & 1,6 & 1,8 \\
\hline Créatine ......... & - & $\longrightarrow$ & 0,1 & 0,8 & 1,2 & 2,3 & 2,5 & 2,9 & 2,5 & 2,2 & 1,9 \\
\hline Azote indéterminé .. & 0 & 27 & 30 & 29 & 26 & 25 & 26 & 30 & 38 & 36 & 46 \\
\hline
\end{tabular}

TABLEAU 4

Évolution de la répartition des constituants azotés du lait de Truie au cours de la lactation. Azote $p$. $100 \mathrm{~g}$ d'azote total

\begin{tabular}{|c|c|c|c|c|c|c|c|c|c|c|c|}
\hline No prélèvement & 0 & 1 & 2 & 3 & 4 & 5 & 6 & 7 & 8 & 9 & 10 \\
\hline $\begin{array}{c}\text { Date de prélèvement } \\
\text { (jours) }\end{array}$ & $\begin{array}{c}0 \\
\text { Partu- } \\
\text { rition }\end{array}$ & 1 & 2 & 7 & 14 & 21 & 28 & 35 & 42 & 49 & Sevrage \\
\hline Caséine & 30,6 & 42,0 & 48,6 & 55,4 & 56,9 & 55,5 & 57,2 & 58,0 & 57,9 & 57,4 & 59,1 \\
\hline Globuline & 38,5 & 28,6 & 17,0 & 12,9 & 12,9 & 14,8 & 15,5 & 15,0 & 14,3 & 15,2 & 17,7 \\
\hline$\beta$-lactoglobuline $\ldots$. & 1,2 & 1,8 & 1,9 & 2,4 & 2,4 & 2,3 & 1,8 & 1,4 & 0,9 & 1,1 & 1,1 \\
\hline Lactalbumine ...... & 16,8 & 16,2 & 16,0 & 15,2 & 14,7 & 14,5 & 14,0 & 14,4 & 14,3 & 11,8 & 10,3 \\
\hline Protéoses-peptones .. & 7,7 & 6,1 & 5,1 & 5,0 & 5,1 & 4,7 & 4,7 & 4,3 & 4,8 & 5,0 & 6,3 \\
\hline Azote non protéique. & 2,3 & 4,1 & 5,4 & 7,6 & 7,8 & 7,1 & 7,1 & 7,3 & 7,9 & 7,3 & 6,9 \\
\hline Urée.......... & 0,3 & 0,6 & 1,0 & 1,7 & 2,2 & 2,1 & 1,7 & 1,5 & 1,2 & 1,2 & 0,8 \\
\hline Ammoniac . & 0 & 0,1 & 0,2 & 0,5 & 0,3 & 0,1 & 0,2 & 0,1 & 0,1 & 0,1 & 0,1 \\
\hline Azote aminé & 1,8 & 1,8 & 2,0 & 2,0 & 2,1 & 2,0 & 2,3 & 2,5 & 2,3 & 2,7 & 2,2 \\
\hline Créatine + crée & - & - & 0,1 & 0,2 & 0,3 & 0,3 & 0,5 & 0,5 & 0,5 & 0,4 & 0,3 \\
\hline Azote non déterminé & 0 & 1,6 & 2,3 & 3,3 & 3,2 & 3,0 & 3,1 & 3,4 & 4,1 & 3,6 & 3,8 \\
\hline
\end{tabular}


- la caséine, principal constituant, évolue sensiblement comme l'azote total : on observe une chute importante du taux au début et une remontée en fin de lactation. C'est avec l'azote non protéique, la seule fraction qui augmente en pourcentage de l'azote total au début de la lactation. Cette augmentation est rapide et, dès la fin de la première semaine, l'équilibre est atteint. Le pourcentage de caséine reste ensuite rigoureusement constant jusqu'à la fin de la lactation.

- Les globulines présentent, en valeur absolue et relative, la baisse de teneur la plus importante pendant les premiers jours de la lactation. Par contre, leur taux remonte moins en fin de lactation que celui de la caséine.

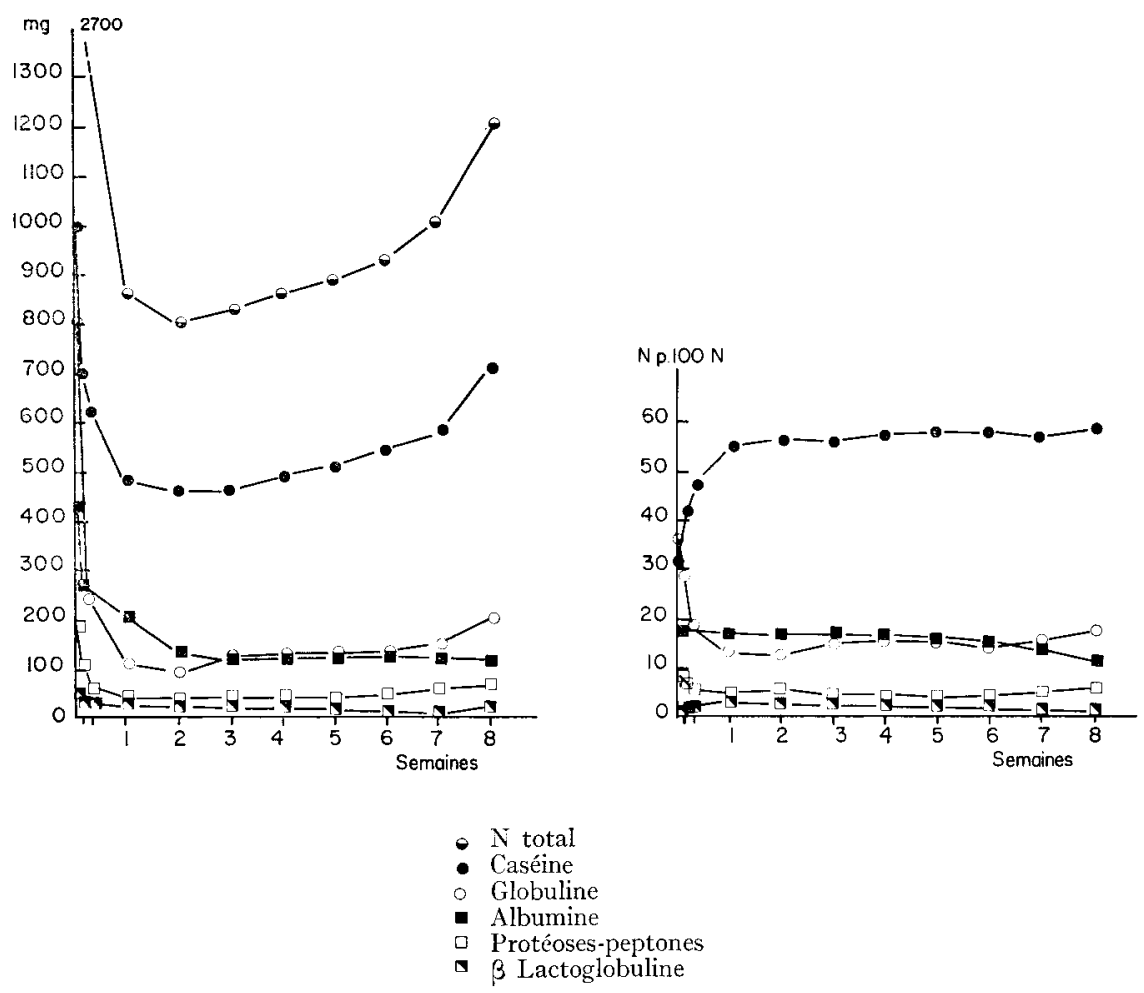

FIG. I. et 2 - Evolution des teneurs en constituants protéiques (en $\mathrm{mg} p$. roo $\mathrm{g}$ de lait frais et en p. 1oo de l'azote total)

Pour tous les constituants, l'évolution est surtout importante au début ou en fin de lactation, mais elle présente des aspects particuliers qu'il convient de souligner pour certains constituants :

- Les albumines constituent la seule fraction qui diminue du début à la fin de la lactation.

- Les constituants non protéiques se répartissent en deux groupes qui présentent des évolutions opposées : les uns comme 1'urée, l'ammoniac, la créatine, et la créatinine augmentent au début de la lactation et diminuent à la fin; les autres 
comme les acides aminés et l'azote non déterminé suivent les variations des protéines : il en résulte qu'au total l'azote non protéique varie peu en valeur absolue et relative,

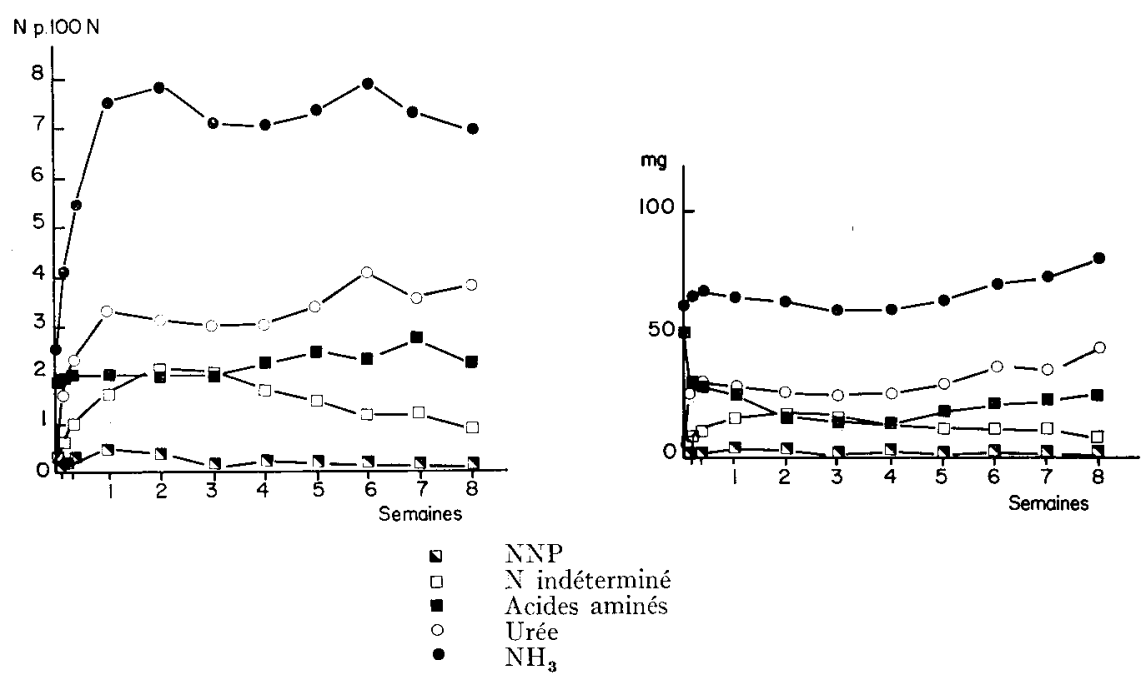

FIG. 3. et 4. - Évolution des teneurs en constituants non protéiques (en $m g$ p. Ioo de lait frais et en p. ioo de l'azote total)

Compte tenu des quantités journalières de lait produites, il résulte de l'évolution des teneurs, que les quantités de chaque constituant secrétées par jour présentent elles-mêmes des variations. Le tableau 5 rapporte quelques-unes des évolutions des

TABLEAU 5

Évolutions des quantités journalières secrétées (g/jour)

\begin{tabular}{|c|c|c|c|c|c|c|c|c|c|c|}
\hline Stade de lactation (j) & 1 & 2 & 7 & $1 / t$ & 21 & 28 & 35 & 42 & 49 & 56 \\
\hline Lait $(\mathrm{kg})$ & 4,3 & 4,7 & 5,5 & 6,0 & 5,7 & 6,2 & 6,5 & 6,2 & 5,5 & 4,7 \\
\hline Azote total $\ldots \ldots \ldots$ & 72,5 & 61,1 & 57,7 & 48,8 & 47,6 & 53,5 & 57,2 & 57,6 & 55,1 & 56,3 \\
\hline Caséine............ & 30,1 & 29,1 & 26,4 & 27,8 & 26,5 & 30,5 & 33,1 & 33,4 & 31,7 & 33,2 \\
\hline Globuline .... & 23,2 & 11,4 & 6,2 & 5,8 & 7,1 & 8,2 & 8,5 & 8,6 & 8,4 & 9,8 \\
\hline Albumines ... & 12,6 & 10,9 & 8,5 & 8,4 & 8,1 & 8,3 & 8,8 & 8,7 & 7,2 & 6,4 \\
\hline Protéoses-peptones ... & 4,4 & 3,0 & 2,4 & 2,9 & 2,3 & 2,5 & 2,5 & 2,8 & 3,4 & 3,1 \\
\hline Azote non protéique.. & 2,9 & 3,2 & 3,7 & 3,8 & 3,4 & 3,8 & 4,3 & 4,4 & 4,0 & 3,8 \\
\hline Urée $\ldots \ldots \ldots \ldots \ldots$ & 0,4 & 0,5 & 0,9 & 1,1 & 1,0 & 0,9 & 0,8 & 0,7 & 0,6 & 0,4 \\
\hline Azote aminé...$\ldots$ & 1,3 & 1,2 & 0,9 & 1,0 & 0,9 & 1,2 & 1,4 & 1,2 & 1,3 & 1,2 \\
\hline
\end{tabular}

quantités sécrétées. On peut ainsi constater que la quantité de caséine varie très peu au cours de la lactation, alors que les albumines sont en diminution constante et que l'azote non protéique a tendance à augmenter. 


\section{C. - Infuence du régime alimentaire}

Le tableau 6 rapporte les teneurs moyennes, pour l'ensemble de la lactation, des principales fractions exprimées en milligrammes d'azote et en p. roo de l'azote total, dans le lait de chacun des lots de truies.

TABLEAU 6

Infuence de l'apport azoté du régime sur les différentes fractions azotées du lait de Truie

\begin{tabular}{|c|c|c|c|c|}
\hline & \multicolumn{2}{|c|}{$\mathrm{N} \mathrm{mg/100}$} & \multicolumn{2}{|c|}{$\mathrm{N}$ p. $100 \mathrm{~g} \mathrm{~N}$} \\
\hline & Lot A & Lot $B$ & Lot A & Lot $B$ \\
\hline 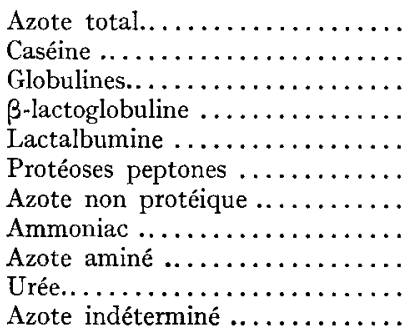 & $\begin{array}{r}993,5 \\
526,8 \\
179,2 \\
21,6 \\
126,4 \\
61,0 \\
69,8 \\
1,8 \\
21,8 \\
12,1 \\
34,1\end{array}$ & $\begin{array}{c}1038,4_{4} \\
566,2 \\
185,6 \\
16,4 \\
151,2\left(^{*}\right) \\
50,6 \\
66,4 \\
1,8 \\
22,1 \\
15,2\left(^{*}\right) \\
27,3\end{array}$ & $\begin{array}{r}100 \\
53,0 \\
17,8 \\
2,2 \\
12,7 \\
6,2 \\
7,0 \\
0,2 \\
2,2 \\
1,2 \\
3,3\end{array}$ & $\begin{array}{r}100 \\
54,6 \\
17,8 \\
1,5 \\
14,6\left(^{*}\right) \\
4,9 \\
6,4 \\
0,2 \\
2,1 \\
1,5\left(^{*}\right) \\
2,7\end{array}$ \\
\hline
\end{tabular}

(*) Différence significative entre les deux lots.

\section{TABLEAU 7}

Infuence de l'apport azoté du régime sur la production laitière de la Truie

\begin{tabular}{|c|c|c|}
\hline & Lot A & Lot $B$ \\
\hline Quantité de lait totale $(\mathrm{kg}) \ldots$ & 301 & 313 \\
\hline Poids moyen des porcelets à 21 jours $(\mathrm{kg}) \ldots$ & 5,1 & 5,0 \\
\hline Poids moyen des porcelets au sevrage $(\mathrm{kg})$.... & 16,2 & 16,7 \\
\hline Nombre de porcelets sevrés $\ldots . \ldots \ldots \ldots \ldots$. & 7,8 & 7,8 \\
\hline $\begin{array}{l}\text { Perte de poids des truies au cours de la } \\
\text { lactation }(\mathrm{kg}) \ldots \ldots \ldots \ldots \ldots \ldots \ldots \ldots \ldots \ldots \ldots\end{array}$ & 16,8 & 17,8 \\
\hline
\end{tabular}

Vu le faible nombre d'animaux dans chaque lot, les différences observées ne sont en général pas significatives, sauf pour la lactalbumine et l'urée. On note cependant une certaine tendance à l'augmentation des teneurs en azote sous l'effet du régime le plus azoté pour les fractions protidiques, à l'exception de la $\beta$-lactoglo- 
buline et des protéoses peptones et pour les fractions non protidiques, à l'exception de 1'azote non déterminé.

On ne note, par ailleurs, aucune différence significative entre les 2 lots en ce qui concerne les quantités de lait sécrétées, les croissances des porcelets et les variations de poids des truies (tabl. 7).

\section{DISCUSSION}

\section{A. - Répartition moyenne}

Sauf en ce qui concerne la tépartition des protéines du sérum dont le total est toutefois le même, nos résultats concordent assez bien avec ceux publiés avant nous par SHEFFY (1952). Les différences que nous observons s'expliquent par l'emploi de

TABLEAU 8

Répartition des fractions azotées du lait chez quelques espèces $N$ p. 100 de $N$ total

\begin{tabular}{|c|c|c|c|c|c|c|c|c|c|}
\hline Espèce & Vache & Chèvre & Jument & Anesse & Femme & Ratte & Baleine & & Truie \\
\hline Auteur & $\begin{array}{l}\text { SHA- } \\
\text { HANI }\end{array}$ & $\begin{array}{l}\text { GaM- } \\
\text { BLE }\end{array}$ & $\begin{array}{l}\text { NeU- } \\
\text { HAUS }\end{array}$ & $\begin{array}{l}\text { ANANTA- } \\
\text { KRISHNAN }\end{array}$ & MaCY & $\operatorname{Cox}$ & $\begin{array}{l}\text { GRE- } \\
\text { GORY }\end{array}$ & SHEFFY & $\begin{array}{c}\text { SALMON- } \\
\text { LEGGAGNEUR }\end{array}$ \\
\hline Année & (1951) & (1939) & $(1960)$ & (1941) & (1961) & (1931) & (1955) & $(1952)$ & (1963) \\
\hline Casëine & 78,5 & 72 & 55 & 39,5 & 33 & 78,2 & $67-52$ & 57,2 & 53,7 \\
\hline Albumine & 6,7 & 13 & & 35,0 & 25 & 4,8 & & 7,4 & 15,5 \\
\hline Globuline & 4,9 & 8 & 45,5 & 2,7 & 12 & 7,8 & $26-44$ & 10,1 & 17,8 \\
\hline $\begin{array}{l}\text { Protéose- } \\
\text { peptone }\end{array}$ & 3,6 & & & & 12 & & & 17,6 & 5,5 \\
\hline $\begin{array}{l}\text { Azote non } \\
\text { protéique }\end{array}$ & 5,9 & 8 & & 22,8 & 17 & 11,9 & $4-6$ & 7,6 & 6,7 \\
\hline Urée & 2,9 & & & 11,0 & 8 & & & 1,3 & 1,3 \\
\hline Azote aminé & 0,9 & & & 3,7 & 2 & & & 2,3 & 2,2 \\
\hline $\begin{array}{c}\text { Azote } \\
\text { indéterminé }\end{array}$ & 1,7 & & & & 7 & & & 3,4 & 2,7 \\
\hline
\end{tabular}

techniques de dosage un peu différentes, notamment en ce qui concerne les albumines et les protéoses-peptones. Ces dernières, en particulier, ont pu faire l'objet d'une surestimation dans le travail de ShEFFy, car il semble bien que la technique de RoWLAND donne des résultats trop élevés pour cette fraction. Par contre, pour les 
fractions non protéiques, où les techniques de dosage ont été sensiblement les mêmes, les résultats de SHEFFy et les nôtres sont particulièrement semblables.

Par comparaison avec le lait des autres espèces, il faut souligner certaines différences, surtout en ce qui concerne les proportions relatives de caséine, d'albumine et de globuline. Nous avons rapporté au tableau 8 les résultats obtenus par quelques auteurs sur le lait de différentes espèces.

On remarquera en particulier que le pourcentage de caséine est beaucoup moins élevé chez la Truie que chez les Ruminants, ou même que chez la Ratte. Par contre, il est plus élevé, ou du même ordre, que ceux des laits de Femme, de Jument, d'Anesse et de quelques espèces sauvages comme la Baleine ou le Rhinocéros (GrzTMEK, I957). Le fait que le rapport protéines du sérum/caséine du lait de Truie soit beaucoup plus élevé que celui de la Vache pourrait éventuellement avoir pour conséquence une meilleure valeur biologique des protéines du lait de Truie. Toutefois, ceci n'a pu être démontré jusqu'à présent, si l'on s'en rapporte à une expérience faite par BRAUDE et al., (I95I) sur des jeunes rats.

Il faut remarquer, par ailleurs, que la répartition des protéines du sérum est assez différente de celle que l'on trouve dans le lait de Vache : c'est ainsi qu'il semble y avoir davantage de globuline que d'albumine dans le lait de Truie et surtout beaucoup moins de $\beta$-lactoglobuline que de lactalbumine, alors que c'est le contraire chez la Vache.

Il est vrai cependant qu'une partie de ces différences peut être inhérente aux méthodes de dosage, qui ne permettent pas d'isoler des fractions pures et peuvent convenir moins bien pour le lait de Truie que pour le lait de Vache.

Il faut noter enfin, que le lait de Truie contient plus d'azote non protéique, en valeur absolue et relative, que le fait de Vache. Ceci est surtout dû aux fractions acides aminés et "azote indéterminé ", car la quantité d'urée est sensiblement la même dans les deux laits. On peut remarquer qu'il en va de même pour d'autres espèces (Femme, Anesse) dont les laits, malgré des différences en azote non protéique total, présentent en valeur absolue, mais non en valeur relative, des concentrations voisines en urée (il en va de même pour la créatine et la créatinine). Ceci semblerait montrer que chez toutes ces espèces la teneur en urée et la teneur en azote total du lait sont indépendantes.

\section{B. - Évolution au cours de la lactation}

Nous distinguerons l'évolution des teneurs des constituants dans le lait, de celle des pourcentages rapportés à l'azote total et de celle des quantités totales sécrétées.

I $^{\circ}$ L'évolution des teneurs des différentes fractions azotées du lait de Truie est assez conforme à celle que 1'on observe chez les autres espèces et confirme les résultats que nous avions déjà trouvés sur la sécrétion des premiers jours de lactation (SALMON-I,EGagneur, I962). E1le est en accord également avec les conclusions de SHEFFy (1952), qui trouve toutefois des teneurs un peu plus élevées que les nôtres pour certaines fractions après un mois, par exemple pour l'azote non protéique.

Dans l'ensemble, l'évolution des différentes teneurs semble obéir à une loi commune : diminution des teneurs, ou dilution, au début de la lactation, stabilité pen- 
dant la période de production constante, puis augmentation des teneurs, ou concentration en fin de lactation à mesure que la quantité de lait sécrétée diminue.

De telles observations rejoignent celles qui ont pu être faites chez la Vache par AzARMe (I938) et par JARRIGE (I957), chez la Brebis par Denamur (I963), chez la Chèvre par Bergman (I937), chez la Femme par MACy (I96I) et par ERICKson (I934). Les seules fractions à échapper à cette règle semblent être l'urée, l'ammoniac, la créatine et la créatinine, dont l'évolution suivent au contraire la courbe de production laitière, c'est-à-dire en fait celle de l'eau. Ce phénomène tendrait donc à appuyer la théorie suivant laquelle ces constituants ne sont pas sécrétés par la mamelle, mais filtrent librement dans le lait, par équilibre avec leur teneur dans les fluides extracellulaires.

$\left.2^{\circ}\right)$ L'évolution relative des différentes fractions montre que si la distribution des protéines dans le lait change très vite au cours des premiers jours de lactation, il n'en est plus ainsi ensuite. C'est ainsi par exemple que la proportion de caséine augmente considérablement au cours de la première semaine, phénomène déjà rapporté par TRAVNICEK (I960), que nous avions nous-mêmes observé (SALMON-LEGAGNEUR, I957), et qui a été aussi signalé chez la Ratte par Glass (I957). Par contre, pendant le reste de la lactation, le rapport $\frac{\text { Caséine }}{\text { Azote total }}$ reste remarquablement constant, ce qui est à rapprocher des observations de JARRIGE (I957) chez la Vache.

Les proportions de globulines et d'azote non protéique total suivent une évolution identique, par contre, les albumines ont un comportement un peu différent: le taux de lactalbumine baisse relativement lentement au début de la lactation, mais d'une façon continue jusqu'à la fin de la lactation. Ceci semblerait indiquer qu'une partie des albumines, pourrait avoir une origine différente des autres protéines du lait, hypothèse généralement admise puisqu'on pense qu'une certaine fraction de ces protéines provient directement du sérum sanguin (BARRY, I96I).

Sur le plan de la valeur nutritionnelle, et mise à part l'évolution des premiers jours, la physionomie de la distribution des protéines du lait de Truie ne varie pratiquement pas au cours de la lactation. Il apparait donc fort improbable que le lait de Truie puisse présenter des variations suffisantes pour que sa valeur biologique en soit modifiée. Il semble bien au contraire, qu'en dehors de la période colostrale, les protéines du lait de Truie conservent une valeur nutritionnelle égale. Les seules modifications possibles sont celles qui proviennent soit d'une augmentation de la teneur en azote total, soit d'une modification du rapport azote/énergie du lait.

$3^{\circ}$ ) L'examen des quantités sécrétées aux différents stades fait aussi apparaître des différences par rapport à ce qui est observé chez la Vache. En effet, pour toutes les fractions azotées du lait de Truie, sauf l'azote non protéique (urée), les quantités sécrétées sont maximales dès le début de la lactation. Par la suite, ces quantités ne font que baisser plus ou moins vite, avec même, semble-t-il, une certaine remontée en fin de lactation pour les globulines et les protéoses-peptones. La caséine est la fraction qui varie le moins et les globulines et les albumines celles qui varient le plus.

Chez la Vache, au contraire, LARson (1957) avait montré que la sécrétion de caséine passait par un maximum au bout de quelques jours puis décroissait d'environ 50 p. Ioo en trois mois. Il en était de même pour la $\beta$-lactoglobuline et la lactal- 
bumine. Par contre, la globuline et les protéoses suivent, chez la Vache, une évolution identique à celle que nous avons rapportées (SANGIORGI, I958).

Peut-on déduire que le mécanisme de la sécrétion de la caséine et des albumines diffèrent chez la Truie et chez la Vache? Certainement pas. Tout au plus peut-on penser que l'évolution des activités sécrétrices n'est pas la même chez ces deux espèces et que la synthèse de la caséine se maintient à son niveau le plus élevé jusqu'à la fin des huit semaines de lactation chez la Truie (ce qui n'est pas le cas pour la lactalbumine).

\section{C. - Infuence du régime alimentaire}

La distribution d'un régime enrichi en azote à des truies semble avoir eu peu d'effet : ni les productions laitières, ni les variations de poids n'ont été modifiées et la richesse azotée du lait n'a été augmentée que de 4 p. Ioo (non significatif), alors que le supplément alimentaire était de $30 \mathrm{p}$. Ioo par rapport au régime normal. Ceci toutefois n'est pas tellement surprenant, compte tenu de ce que le régime normal apportait a priori suffisamment d'azote pour couvrir les besoins normaux de la lactation et qu'une amélioration paraissait donc peu probable. Ce résultat concorde également avec ceux qui ont pu être obtenus en d'autres circonstances, ou sur d'autres espèces. Il est à rapprocher de ceux de BOWLAND (I949) et SHEFFy (I952) également sur la Truie, de ceux de Deb (I962) et de Karmarkar (I959) sur la Femme, de ceux de PERRIN (I958) sur la Brebis et des nombreux travaux réalisés sur ce point sur la Vache, comme ceux de Perkins (I940), Breirem (i949), Stein (i957), Woodman (I957), Rook (I959) et Kirmeier (I960). 'Tous ces travaux montrent, en effet, que toute modification du niveau alimentaire, ou du niveau azoté, n'entraîne pas en général de variation importante de la quantité d'azote sécrétée dans le lait.

Par contre, il est assez fréquent que certains auteurs signalent une modification de teneur de 1'un ou l'autre des constituants azotés sous l'effet de certains régimes. Pour Rook (I959), KaTMARKar (I959) et Bowland (I949), les modifications portent surtout sur les protéines et notamment sur la caséine ; pour DEB (I962), PERKINS (I930) et StEin (I957), seul l'azote non protéique s'élève lorsque la teneur en azote du régime croît. Dans notre expérience, deux fractions ont été augmentées d'une façon significative par le régime hyperazoté : la lactalbumine et l'urée ; deux autres ont montré une légère tendance à l'augmentation : la caséine et la globuline; les autres ont été diminuées.

Ces divergences de résultats peuvent, peut-être, s'expliquer en fonction des niveaux alimentaires utilisés : lorsque le niveau de référence est bas, une amélioration du régime peut stimuler la sécrétion laitière et donc favoriser la production d'une plus grande quantité de protéines spécifiques, c'est-à-dire essentiellement de la caséine et des globulines. Lorsque le niveau est élevé, le supplément azoté n'a pas d'effet sur la synthèse de la caséine, mais il peut en exercer une sur la protéinémie du sang et donc sur les teneurs en albumine et en urée du lait, dans la mesure où celles-ci proviennent directement du sang. Dans notre cas, le niveau alimentaire de référence était haut, et c'est essentiellement ces fractions qui ont été affectées.

Dans l'ensemble donc, il ne semble pas qu'on puisse faire état ici de différences quantitative ou qualitative liées à l'alimentation, propres à l'espèce porcine, alors 
qu'il n'en va pas de même dans d'autres secteurs, notamment celui des lipides (SALMON-LEGAGNEUR, I962).

Il ressort donc de cette étude que c'est plus par la physionomie de ses protéines (teneurs élevées en lactalbumine et surtout en globuline, mais relativement faibles en caséine et en $\beta$-lactoglobuline) et l'évolution particulière de certaines fractions au cours de la lactation (caséine, lactalbumine, urée), que semble se caractériser essentiellement la matière azotée du lait de Truie.

Rę̧u pour publication en décembre 1963 .

\title{
SUMMARY
}

\author{
NITROGEN CONSTITUENTS OF SOW'S MILK : \\ EFFECT OF STAGE OF LACTATION AND LEVEL OF FEEDING
}

A detailed study of the main protein and non-protein nitrogen fractions of sow's milk has been carried out during the lactation of $\mathrm{I} 2$ sows with 2 levels of dietary protein (I4 and I9 P. IOO).

$\left.\mathrm{I}^{\circ}\right)$ The mean values for the nitrogen distribution of sow's milk, expressed as $\mathrm{mg}$ nitrogen $/ \mathrm{roo} \mathrm{g}$ milk, were : total I or 4 , casein 545 , globulin 18 I, $\beta$-lactoglobuline 19 , lactalbumin 138 , proteosepeptone 56 , non protein nitrogen 68, urea $\mathrm{I}_{4}$, amino acids $22, \mathrm{NH}_{3} \mathrm{I}, 7$, creatine 3,5 , creatinine $\mathrm{I}, 9$ and unaccounted 23. In terms of proportion of total nitrogen, sow's milk is richer than cow's milk in lactalbumin, globulin and proteose-peptone, and less rich in casein, $\beta$-lactoglobuline and urea. Individual variations were considerably lower for casein than for serum protein.

$\left.2^{\circ}\right)$ Percentages of most of the constituents dropped sharply at the beginning of the lactation and slightly increased towards the end (but it was the reverse for urea and creatine). Therefore the quantities secreted daily, particularly of casein, were nearly constant throughout the lactation. Casein, $\beta$-lactoglobulin and non-protein nitrogen increased as a proportion of total nitrogen during the first week of lactation but did not vary thereafter.

$3^{\circ}$ ) Increasing the nitrogen content of the ration had only a small effect on milk composition. Only the lactalbumin and urea contents increased significantly, by about 20 p. 100 .

\section{RÉFÉRENCES BIBLIOGRAPHIQUES}

Anantakrishnan C. P., I94I. Studies on Ass's milk. J. Dairy Res., 12, I I9-I zo.

Aschaffenburg R., Drewry J., 1959. New procedure for the routine determination of the various noncasein proteins of milk. $X V$ intern. Dairy Congr., 3, I63I-I637.

Azarme E., 1938. Variations in the protein content of milk during lactation. J. Dairy Res., 9, 12 I-I 52.

Barry J. M., I961. Protein metabolism, in Kon S. K., Cowie A. T. Milk. vol. II, 389-419, Academic Press, N. Y.

Bergman A. J., Turner C. W., 1937. The composition of the colostrum of the dairy goat. J. Agric. Sci., 20, 37-45.

Braude R., Henry K. M., Kon S. K., Mitchell K. G., i95i. Nutritive value for rats of sow's milk in comparison with cows' milk. J. Dairy Res., 18, $283^{-290 .}$

Bowland J. P., Grummer R. H., Phillips P. H., Bohstedt G., I949. The effect of the plane of nutrition on the composition of sow's colostrum and milk. J. Anim. Sci., 8, r99-205.

Breirem K., 1957. Nutrition and lactation in domestic animals and particularly in the cow. Ann. Nutr. Alim., 11, $\mathrm{A}_{3}-\mathrm{A}_{3}$.

Cox W. M., Mueller A. J., I937. The composition of milk from stock rats and an apparatus for milking small laboratory animals. J. Nutr., 13, 249-26r.

DEb A. K., Cama H. R., I962. Studies on human lactation. Dietary nitrogen utilization during lactation and distribution of nitrogen in mathers milk. Brit. J. Nutr., 16, 65-73. 
Denanilr R., 1963. Résultats non publiés.

Denis W., Minot A. S., I919. The methods for the quantitative determination of the non-protein nitrogenous constituants of milk. J. Biol. Chem., 37, 353-346.

Erickson 13. N., Gulick M., IIuncher II. A., Macy J. G., I934. Human milk studies. XV. The nonprotein nitrogen constituants. J. Biol. Chem., 106, I45-I60.

Gamble J. A., Ellis N. R., Bresley A. K., I939. Composition and properties of goats milk as compared with cows milk. U.D.S. A. tech. Bull., 67 r.

Glass R. L., 1957. Chemical, physical, and biological studies of rat's milk and its componants. Diss. Abstr., $17,494-495$.

Gregery M. E., Kon K. S, Rowland S. J., Thompson S. Y., 1955. The composition of the milk of the blue whale. J. Dairy Res., 22, 108-1 I2.

Grzimek B., 1957. Die Zusammensetzung der Nashornmilch. Millhwissenschaft, 12, 23 I.

IIEnry K. M., 1957. The nutritive value of milk proteins. Dairy Sii. Abstr., 19, 603-5r6.

JARRIGE R., I957. Études sur les variations de la richesse en constituants azotés des laits de Vache. II. Evolution au cours de la lactation et de la gestation. 1 inn. Zootech., 6, 4I-80.

Karmarkar M. G., Choksi H. R., Shail V. K., i959. Studies on human lactation. J. Pediat., 55, 48I484 .

Kiermeier F., Renner F., Ig60. Einfluss des Futterung auf den Eiweissgehalt der Milch. Z. Tierphysiol. Tievernäh. Futterm., 15, 332-3+3.

Larson B. L., Kendall K. A., I957. Protein production in the Bovine. Daily production of the specific milk proteins during the lactation period. J. Dairy Sci. 40, 377-385.

MaCy I. G., Kelly 11. J., i96I. Human milk and cow's milk in infant nutrition, in Kon S. K., CowiEA. T. Milk, vol. II, 265-303, Acad. Press X. Y.

Nevinaus U., I960. Entersuchungen und Beobachtungen über gewinnung, Eirrenschaften und für dic Ernährung menschlicher Sä̈glinge. Zuchtungskunde, 32, 513-520.

Neuhaus U., 1961. Die Milcheistung der Sau und die Zusammensetzung und Eigenschaften der Sauenmilch. Z. Tierzucht. Zuchtungsbiol., 75, 160-191.

Perkins A. E., 1930. Protein studies. Ohio Agr. Exp. Stat. Bull., 446, i I 5-1 I6.

P'ERRIN D. R., 1958. The chemical composition of the colostrum and milk of the ewe. J. Dairy Res., 25, $70-74$.

Rook J. A. F., RowtAND S. J., 1959. Fiffect of spring grazing on the constituents of the solids-not-fat of milk. $X I V$ intern. Dairy Congr., 1, 20+-zog.

Rowland S. J., 1933. The determination of the nitrogren distribution in milk. J. Lairy Res., 9, 42-46.

Salmon-Legagneur E., $195^{5}$. La mesure de la production laitière de la Truie. Ann. Zootech., 5, 345-352.

Salmon-Leragneur li, i959. Description et utilisation d'une machine à traire les truies. Ann. Zootech., 8, $345^{-35}$.

Salmon-Legagneur le., Aumatre A., i 962 . Infuence de la quantité de lait et de sa composition sur la croissance du porcelet sous la mère. Ann. Zootech., 11, isi-ig6.

Salmon-Legagneur E., Guiguen L., 1962. Évolution de la composition chimique du colostrum chez la Truie. Ann. Biol. anim. Bioch. Biophys., 2, 229-317.

SALMON-I,EgAGNEUR E., ig62. Influence de l'introduction de graisse dans la ration sur la production et la composition du lait de Truie. Ann. Zootech., 11, 295-309.

SANG1ORgI A., I958. Variations dans la répartition électrophorétique des protéines du sérum de lait de Vache au cours de la lactation. Reo. Clin. Pediat., 61, 106-1 I3.

SHahani K. M., Sommer H. H., 195I $a$. The protein and non protein nitrogen fractions in milk. I. Methods analysis. J. Dairy Sci., 34, 1003-1009.

Sialiani K. M., Sommer H. H., 195 I $b$. The protein and non protein nitrogen fractions in milk. II. Their content in fresh raw milk. J. Sci., 34, 1010-1013.

Sheffy B. E., Shainane K. M., Grummer R. H., Phillips P. H., Sommer H. H., 1952. Nitrogen constituonts of sow's milk as affected by ration and stage of lactation. J. Nutr., 48, I03-II4.

SiRRY L., HASSAN H. A., I954. Composition of goat milk in Lgypt. Indian J. Dairy Sci., 7, I88-193.

Stein J. F., i957. Some effects of feeding excess protein to lactating dairy cows. Diss. Abstr., 17, 936-937.

Travnicek J., 1950. La commosition chimique du colostrum et du lait des truies Large White (Tchèque) Ann. Acal. Tchecosl. Agric., 33, 497-512.

Woodman I1. E., 1957. Rations for livestock. Bull. Min. Agric. Fish. Fd, London, i4 éd., 48, $5^{\text {I }}$ p. 